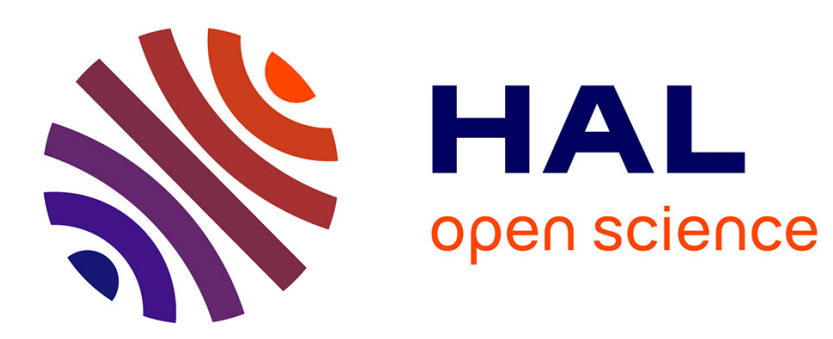

\title{
Reliability-based analysis and design of foundations resting on a spatially random soil
}

\author{
Abdul-Hamid Soubra, Dalia S Youssef Abdel Massih
}

\section{To cite this version:}

Abdul-Hamid Soubra, Dalia S Youssef Abdel Massih. Reliability-based analysis and design of foundations resting on a spatially random soil. ASCE Geocongress 2008, 2008, Nouvelle-Orléans, United States. hal-01008820

\author{
HAL Id: hal-01008820 \\ https://hal.science/hal-01008820
}

Submitted on 22 Sep 2018

HAL is a multi-disciplinary open access archive for the deposit and dissemination of scientific research documents, whether they are published or not. The documents may come from teaching and research institutions in France or abroad, or from public or private research centers.
L'archive ouverte pluridisciplinaire HAL, est destinée au dépôt et à la diffusion de documents scientifiques de niveau recherche, publiés ou non, émanant des établissements d'enseignement et de recherche français ou étrangers, des laboratoires publics ou privés. 


\title{
Reliability-based analysis and design of foundations resting on a spatially random soil
}

\author{
Abdul-Hamid Soubra ${ }^{1}$ and Dalia S. Youssef Abdel Massih ${ }^{2}$ \\ ${ }^{1}$ Professor, University of Nantes, GeM, UMR CNRS 6183, Bd. de l'université, BP 152, 44603 Saint- \\ Nazaire cedex, France. E-mail: Abed.Soubra@univ-nantes.fr \\ ${ }^{2}$ PhD Student, University of Nantes \& Lebanese University, BP 11-5147, Beirut, Lebanon. E-mail: \\ Dalia.Youssef@univ-nantes.fr
}

\begin{abstract}
This paper presents the effect of the spatial variability of the soil shear strength parameters on the reliability analysis and design of a vertically loaded shallow strip footing against bearing failure. The deterministic model used is based on the upper-bound method of limit analysis. The Hasofer-Lind reliability index based on the most critical probabilistic failure surface is calculated for the assessment of the footing reliability. The random fields used in the analysis are the soil shear strength parameters. Normal and non-normal anisotropic random fields with or without cross correlation are considered. The two random fields are averaged along the potential slip lines of the failure mechanism. It was found that the assumption of negative cross correlation, soil heterogeneity and anisotropy regarding the autocorrelation distance gives a higher reliability index than the hypothesis of no cross correlation, homogeneous and isotropic soil respectively. These assumptions merely allow the reliability to be more accurately estimated and thus lead to an economic design. The failure probability was found more sensitive to the variability of the angle of internal friction than the cohesion. For design, an iterative procedure is performed to determine the breadth of the footing for a target failure probability.
\end{abstract}

\section{INTRODUCTION}

Several authors have investigated the reliability-based analysis of foundations against bearing failure. Some authors have modelled the uncertainties of the different parameters as random variables (e.g. Low and Phoon, 2002) without introducing the spatial variability of the soil parameters. Others (Cherubini 2000) have considered the effect of the soil spatial variability (i.e. soil heterogeneity) by using a simplified approach. Later on, several authors (Griffiths et al. 2002; Fenton and Griffiths 2003 and Popescu et al. 2005 among others) have modelled the uncertain soil parameters more rigorously as random processes and have examined the effect of the spatial variability of these parameters on the ultimate bearing capacity by using finite elements codes. However, most of these studies (except that of Fenton and Griffiths 2003) consider only a single random process in their analysis and require high computation time due to the use of Monte Carlo simulations. 
In this paper, the effect of the soil spatial variability on the reliability analysis and design of a vertically loaded strip foundation is presented. Two random processes concerning the soil cohesion and angle of internal friction are used in the analysis. The two random fields are assumed to be anisotropic with different values of vertical and horizontal autocorrelation distances. The punching mode of the ultimate limit state is analysed. A limit analysis model based on the non-symmetrical multiblock failure mechanism presented by Soubra (1999) is used here. This model is rigorous and has the advantage of being more efficient than the commonly used finite element approach which requires much more computation time. In this model, the random fields are averaged along the different potential slip lines of the failure mechanism. After a brief description of the basic concepts of spatial averaging, reliability index and failure probability, the probabilistic model and the corresponding numerical results are presented and discussed.

\section{BASIC RELIABILITY CONCEPTS}

\section{Spatial averaging of a random field}

The average value of a random field $Z(x, y)$ over a domain $A$ is given by:

$$
Z_{A}=\frac{1}{A} \cdot \int_{A} Z(X) \cdot d X
$$

If the random field is averaged over a one-dimensional domain as for the slip lines of the failure mechanism used in this paper ( $c f$. Figure 1), the domain $A$ will correspond to the distance $L$ of the segment along which a local average of the random field is defined. By averaging the random field over two arbitrary situated segments $L_{i}$ and $L_{j}$, two variables representing two local averages are found accordingly to equation (1) and a correlation may exist between these variables. This correlation is calculated by averaging the correlation between the random variables at all points on both segments. It is given by (Knabe et al. 1998):

$$
\rho\left(L_{i}, L_{j}\right)=\frac{1}{L_{i} L_{j}} \int_{0}^{L_{i}} \int_{0}^{L_{j}} \rho(u) d s d l
$$

where $\mathrm{u}$ is the distance that separates any two points of the two segments $L_{i}$ and $L_{j}$.

\section{Reliability index and failure probability}

The Hasofer-Lind reliability index is defined by:

$$
\beta_{H L}=\min _{G(x) \leq 0} \sqrt{\left(\frac{x-\mu}{\sigma}\right)^{T}[R]^{-1}\left(\frac{x-\mu}{\sigma}\right)}
$$

in which $x$ is a random variable, $\mu$ and $\sigma$ are the mean and standard deviation of $\mathrm{x}$, $(x-\mu / \sigma)$ is the vector the $n$ centred and reduced random variables, $R$ is the correlation matrix and $F$ is the failure region. The minimisation of (3) is performed subject to the constraint $G(x) \leq 0$ where the limit state surface $G(x)=0$, separates the $n$ dimensional domain of random variables into two regions: a failure region $F$ 
represented by $G(x) \leq 0$ and a safe region given by $G(x)>0$. An intuitive interpretation of the reliability index was suggested in Low and Tang (1997) where the concept of an expanding ellipse led to a simple method of computing the HasoferLind reliability index in the original space of the random variables for both normal and non-normal variables with or without correlation. The method of computation of the reliability index using the concept of an expanding ellipse suggested by Low and Tang (1997) is used in this paper. From the Hasofer-Lind reliability index $\beta_{H L}$, one can approximate the failure probability by using the First Order Reliability Method FORM as follows:

$P_{f} \approx \Phi\left(-\beta_{H L}\right)$

where $\Phi(\cdot)$ is the cumulative distribution function of a standard normal variable.

\section{RELIABILITY ANALYSIS OF A STRIP FOUNDATION ON A SPATIALLY RANDOM SOIL MEDIUM}

In this paper, the effect of the spatial variability of the soil shear strength parameters on the reliability analysis and design of a strip foundation subjected to a central vertical load is presented. The cohesion $\mathrm{c}$ and the angle of internal friction $\varphi$ are considered as random fields. A deterministic model based on the upper-bound method of limit analysis is used to study the punching failure mode of the ultimate limit state. Due to the soil heterogeneity, the non-symmetrical multiblock failure mechanism presented by Soubra (1999) is adopted ( $c f$. Figure 1).

\section{Failure mechanism}

The present failure mechanism is composed of a sequence of $n$ triangular rigid wedges. It is described by $2 n$ angular parameters $\left[\alpha_{i}(i=1, \ldots, n), \beta_{i}(i=1, \ldots, n)\right] . V_{i}$ and $V_{i, i+1}$ are respectively the velocity of block $i$ and the inter-block velocity between blocks $i$ and $i+1$. The first wedge $\mathrm{ABC}$ is translating as a rigid body with a downward velocity $V_{1}$ inclined at an angle $\varphi_{o 1}$ to the discontinuity line AC. Note that the foundation is assumed to move with the same velocity as that of the first block (i.e. $V_{1}$ ). The wedge $i$ is assumed to move with a velocity $V_{i}$ inclined at $\varphi_{o i}$ to line $d_{i}$ (cf. Figure 1) where $\varphi_{o i}$ is the average value of the random field of the angle of internal friction along the line $d_{i}$. The inter-block velocity $V_{i, i+1}$ is inclined at $\varphi_{r i}$ to line $l_{i}$ where $\varphi_{r i}$ is the average value of the random field of the angle of internal friction along the segment $l_{i}$. As for the angle of internal friction, the random field of the cohesion is averaged along each line of the mechanism; $c_{o i}$ is the average value along the line $d_{i}$ and $c_{r i}$ is the average value along the line $l_{i}$. The calculation of ultimate bearing capacity of the footing is performed by equating the total rate at which work is done by the foundation load, the soil weight in motion and the ground surface surcharge to the total rate of energy dissipation along the lines of velocity discontinuities of the failure mechanism. 


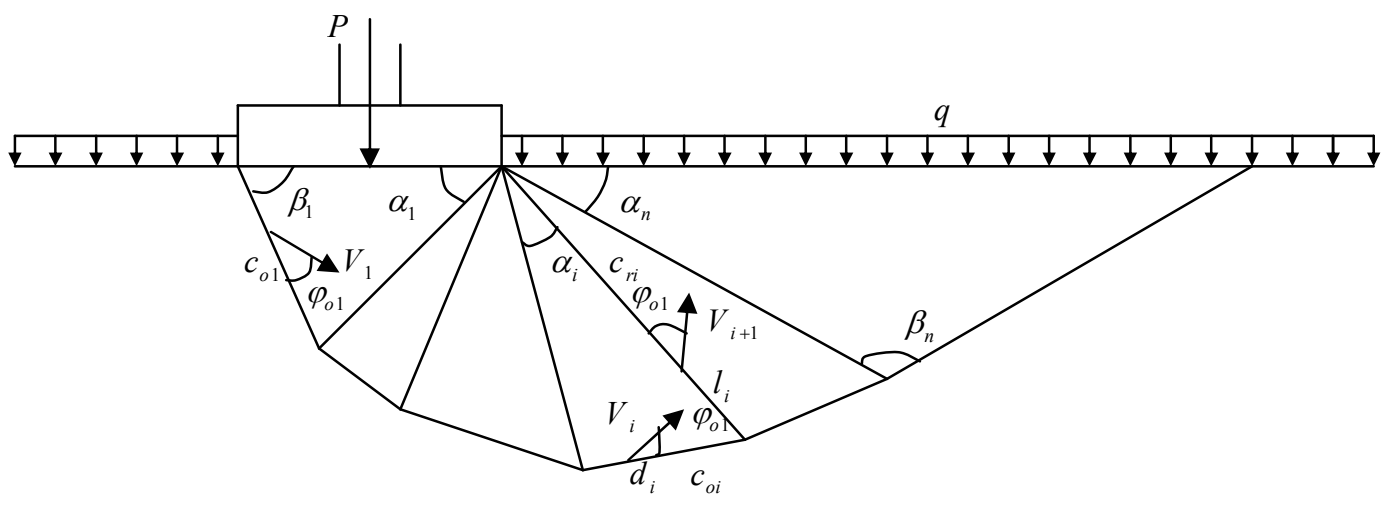

FIG. 1. Non-symmetrical multiblock failure mechanism

\section{Performance function, autocorrelation function and reliability index}

The performance function used in the reliability analysis is defined with respect to the punching failure mode of the soil. It is given as follows:

$G=\frac{P_{u}}{P_{S}}-1$

where $P_{u}$ is the ultimate foundation load and $P_{S}$ is the vertical applied load.

An anisotropic autocorrelation function is used in this paper for both the cohesion and the angle of internal friction. It is given by an exponential first order function as follows (e.g. Vanmarcke, 1983):

$\rho(\delta x, \delta y)=e^{-2 \sqrt{\left(\frac{\delta x}{D_{h}}\right)^{2}+\left(\frac{\delta y}{D_{v}}\right)^{2}}}$

where $D_{h}$ and $D_{v}$ are the autocorrelation distances in the horizontal and vertical directions respectively and, $\delta x$ and $\delta y$ are the lag distances in the horizontal and vertical directions respectively. A constant cross-correlation $\rho_{c, \varphi}$ is used here.

The Hasofer-Lind reliability index given by equation (3) is used for the computation of the reliability of the foundation. The vector of random variables $\mathrm{x}$ in this equation is composed of the local average values of the soil shear strength random fields. Consequently, the reliability index depends on $4 n-2$ random variables $\left(c_{r j}, \varphi_{r j}, c_{o i}, \varphi_{o i}\right)$ with $i=1, \ldots, n$ and $j=1, \ldots, n-1$. The correlation matrix [R] is a square matrix of dimensions $(4 n-2) \times(4 n-2)$ in which $(2 n-1)^{2}$ components are determined by equation (2) using numerical integration. Each component represents the local average correlation between two average values of the random field along two different lines of the failure mechanism. Others $(2 n-1)^{2}$ components correspond to the value of the cross-correlation of the two random fields. The numerical integration is performed using the Gauss-Legendre quadrature method. 


\section{NUMERICAL RESULTS}

The numerical results presented in this paper consider the case of a shallow strip foundation with breadth $B=2 \mathrm{~m}$ subjected to a vertical load. The soil has a unit weight of $18 \mathrm{kN} / \mathrm{m}^{3}$. No surcharge loading $(q=0)$ is considered in the analysis. The illustrative values used for the statistical moments of the shear strength random fields are as follows: $\mu_{c}=20 \mathrm{kPa}, \mu_{\varphi}=30^{\circ}, C O V_{c}=20 \%, C O V_{\varphi}=10 \%$. For the probability distributions of the random fields, two cases are studied. In the first case, referred to as normal random fields, $\mathrm{c}$ and $\varphi$ are considered as normally distributed random fields. In the second case referred to as non-normal random fields, c follows a log-normal distribution while $\varphi$ is assumed to be bounded and a beta distribution is used. For both cases, uncorrelated (i.e. $\left.\rho_{c, \varphi}=0\right)$ or negatively correlated $\left(\rho_{c, \varphi}=-0.5\right)$ random fields are considered.

\section{Probabilistic failure surface}

A common approach to determine the reliability of a stressed soil mass is based on the calculation of the reliability index $\beta_{H L}$ corresponding to the deterministic failure surface. In this paper, a more rigorous approach is used. It consists in the determination of the reliability index by minimizing the quadratic form of equation (3) not only with respect to the values of the local averages but also with respect to the geometrical parameters of the failure mechanism $\left(\alpha_{i}, \beta_{i}\right)$. Notice that the correlation matrix [R] should be calculated for each function evaluation during the minimization process. This is because of the change in the potential failure mechanism. Thus, $(2 n-1)^{2}$ numerical integrations are required for each function evaluation. This approach leads to a much higher computation time than calculating the reliability index using the deterministic surface. A comparison of the reliability index and the corresponding critical mechanism as obtained by the two approaches is presented in figure (2) for $P_{S}=1000 \mathrm{kN} / \mathrm{m}, D_{h}=10 \mathrm{~m}, D_{v}=2 \mathrm{~m}, \rho_{c, \varphi}=0$ and $\mathrm{n}=10$. The minimization of the quadratic form of equation (3) is performed with respect to $(6 n-2=58)$ parameters $\left(\alpha_{i}, \beta_{i}, c_{r j}, \varphi_{r j}, c_{o i}, \varphi_{o i}\right)(i=1, \ldots, n-1$ and $j=1, \ldots, n)$. The surface corresponding to the minimum reliability index is referred to here as the critical probabilistic surface. The reliability index calculated with respect to the critical probabilistic surface is smaller (i.e. more critical) than the one calculated using the critical deterministic surface ( $c f$. Figure 2).

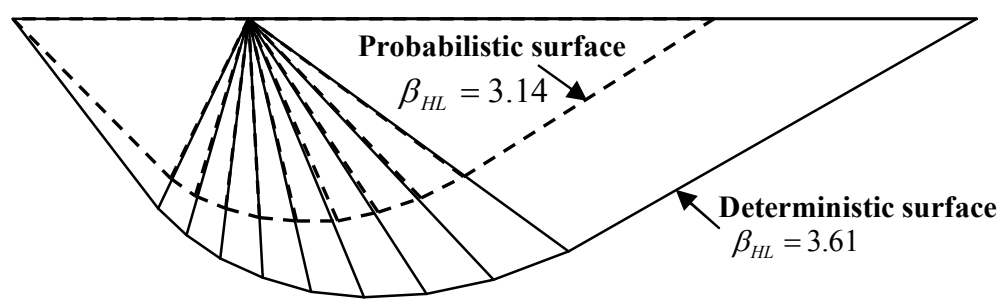

FIG. 2. Deterministic and probabilistic failure surfaces 
It was found that the probabilistic failure surface is significantly sensitive to a variation of the applied load and nearly insensitive to the variation of the coefficient of variation, the autocorrelation distances and the cross-correlation of the soil properties. Thus, in the next sections, only one probabilistic failure surface will be calculated for a given applied load (i.e. a given safety factor) and given values of $\mu_{c}$ and $\mu_{\varphi}$. This significantly reduces the computation time of the minimization process.

\section{Variation of the reliability index with the statistical parameters of the shear} strength properties

Figure (3) shows the variation of the reliability index with the autocorrelation distance for normal and non-normal isotropic fields $\left(D=D_{v}=D_{h}\right)$. Both uncorrelated and negatively correlated random fields are considered. A safety factor $F=3$ is used. It can be shown that the reliability index corresponding to uncorrelated fields is smaller than the one of negatively correlated fields. One can conclude that assuming negatively correlated shear strength parameters gives economic design in comparison to assuming uncorrelated ones. For the case in hand, the results of normal and non-normal fields are nearly identical. One can also notice that for a high soil heterogeneity corresponding to small values of $D / B$, one obtains a high reliability index which means that the assumption of soil heterogeneity increases the reliability of the foundation and thus leads to economic designs.

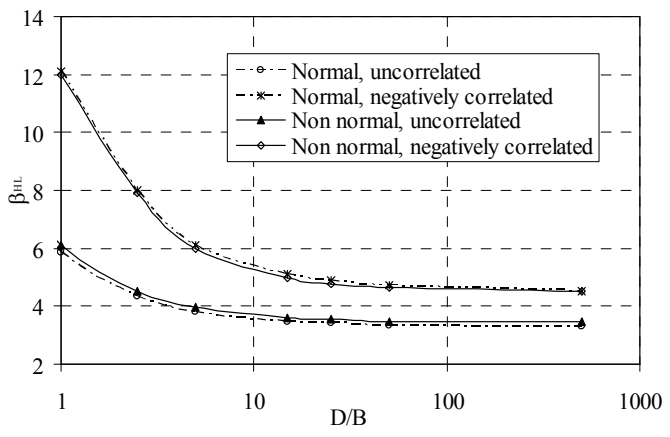

FIG. 3. Effect of the probability

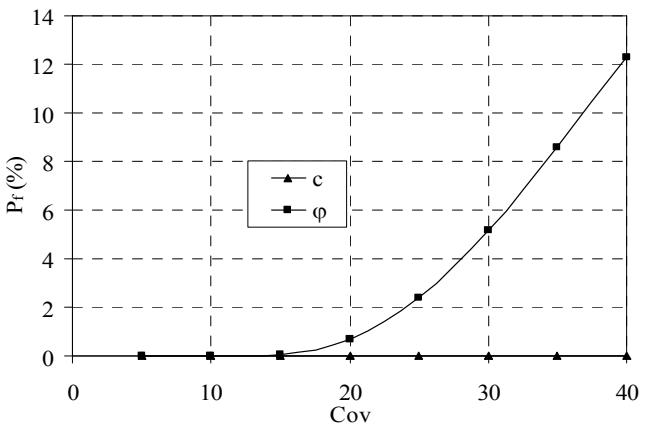

FIG. 4. $P_{f}$ versus $\mathrm{COV}_{\mathbf{c}}$ and $\mathrm{COV}_{\varphi}$ distribution and cross-correlation on $\beta_{H L}$

\section{Sensitivity of the failure probability to the variability of the soil shear strength}

Figure (4) shows the FORM failure probability versus the coefficient of variation of $\mathrm{c}$ or $\varphi$. For each curve, the coefficient of variation of a random field is hold to the same constant value given in the introduction of the section "Numerical results" and the coefficient of variation of the second field is varied over the range $5 \%-40 \%$. A safety factor $F=3$ is used. Anisotropic non-normal random fields with no cross correlation $\left(D_{h}=10 m, D_{v}=2 m, \rho_{c, \varphi}=0\right)$ are considered. The results show that the failure probability is highly influenced by the coefficient of variation of the angle of internal friction, the greater the scatter in $\varphi$ the higher the failure probability of the foundation. This means that the accurate determination of the statistical properties of this parameter is very important in obtaining reliable probabilistic results. In contrast, the coefficient of variation of $\mathrm{c}$ does not significantly affect the failure probability. 


\section{Influence of the anisotropy of the soil shear strength random fields}

In general, the variability of the soil in the horizontal direction is different from that in the vertical direction. Figure (5) shows the variation of the failure probability with the ratio $D_{v} / D_{h}$ for $D_{h}$ varying between 0.1 and $100 \mathrm{~m}$. One can conclude from this figure that for the practical case $D_{v} / D_{h}<1$ for which the variability in the vertical direction is higher than that in the horizontal direction, the reliability index is underestimated if the calculation is performed using the assumption of isotropic fields (i.e. $D_{v} / D_{h}=1$ ) and leads to non-economic design. When both autocorrelation distances highly increase, the reliability index tends to the value obtained when modeling the soil shear strength parameters as random variables.

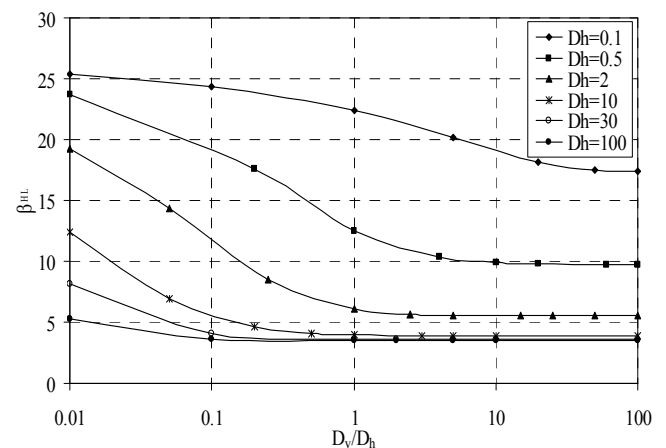

FIG. 5. Influence of anisotropy on the reliability index

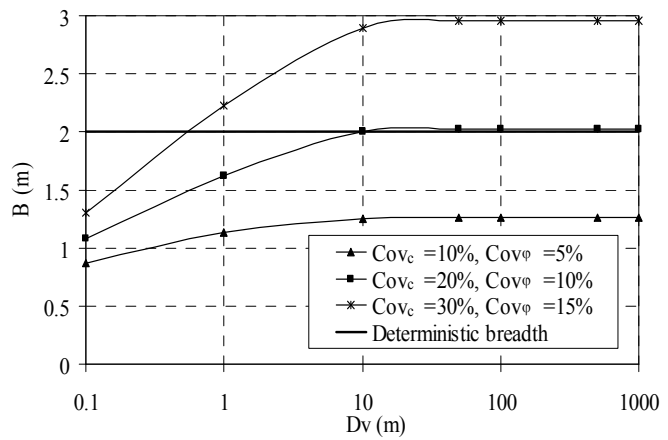

FIG. 6. Comparison between probabilistic and deterministic design

\section{Probabilistic design}

The conventional approach used in the design of a shallow foundation is to prescribe a target safety factor (generally $F=3$ ) and to determine the corresponding breadth $\mathrm{B}$ of the footing. Recently, a reliability-based design $(R B D)$ has been used by several authors (e.g. Low 2005) on the case of a homogeneous soil. In this paper, a $R B D$ is used for spatially varying soil. The approach consists in the calculation of the footing breadth B for a target reliability index of 3.8 as suggested by Eurocode 7 for the ultimate limit states. This foundation breadth is called hereafter "probabilistic foundation breadth". Figure (6) presents the deterministic and the probabilistic foundation breadth for different values of the coefficients of variation of the shear strength random fields and their vertical autocorrelation distance. Anisotropic ( $D_{h}=10 \mathrm{~m}$ ) non-normal random fields with no cross correlation are considered. This figure also presents the deterministic breadth corresponding to a safety factor of 3 . It can be noticed that the probabilistic foundation breadth increases with the increase of $D_{v}$ and the increase of the coefficients of variation of the shear strength random fields. The assumption of isotropic random fields (i.e. $D_{v}=D_{h}=10 \mathrm{~m}$ ) gives higher foundation breadth in comparison to the practical case of anisotropy corresponding to higher horizontal autocorrelation distance (i.e. $D_{v}<D_{h}$ ). As a conclusion, the deterministic footing breadth may be greater or smaller than the probabilistic foundation breadth, depending on the soil variability. 


\section{CONCLUSION}

This paper presents the effect of the soil shear strength spatial variability on the reliability analysis and design of a shallow strip footing against bearing failure. The deterministic model used is based on the upper-bound method of limit analysis. The Hasofer-Lind reliability index and the FORM failure probability were calculated. The random fields used in the analysis are the soil shear strength parameters. Normal and non-normal anisotropic random fields with or without cross correlation are considered. It was found that the reliability index calculated with respect to the critical probabilistic surface is smaller (i.e. more critical) than the one determined using the critical deterministic surface. The probabilistic failure surface is significantly sensitive to a variation of the applied load (i.e. safety factor) and nearly insensitive to the variation of the coefficient of variation, the autocorrelation distances and the cross-correlation of the soil properties. The failure probability is more sensitive to the variability of the angle of internal friction than the cohesion. For the practical case for which the variability in the vertical direction is higher than that in the horizontal direction, the reliability index is underestimated if the calculation is performed using the assumption of isotropic fields and leads to non-economic design. Finally, the deterministic footing breadth may be greater or smaller than the probabilistic foundation breadth, depending on the soil variability.

\section{REFERENCES}

Cherubini, C. (2000). "Reliability evaluation of shallow foundation bearing capacity on $c^{\prime}$, $\varphi^{\prime}$ soils." Can. Geotech. J., 37, 264-269.

Fenton, G. A., and Griffiths D. V. (2003). "Bearing capacity prediction of spatially random c- $\varphi$ soils." Can. Geotech. J., 40, 54-65.

Griffiths, D. V., Fenton, G. A., and Manoharan, N. (2002). "Bearing capacity of rough rigid strip footing on cohesive soil: Probabilistic study." J. of Geotech. \& Geoenv. Engrg., ASCE, 128(9), 743-755.

Knabe, W., Przewlocki, J. and Rozynski, G. (1998). "Spatial averages for linear elements for two-parameters random field." Prob. Engng. Mech. 13(3), 147-167.

Low, B. K. (2005). "Reliability-based design applied to retaining walls." Géotechnique, 55(1), 63-75.

Low, B. K., and Phoon, K. K. (2002). "Practical first-order reliability computations using spreadsheet." Probabilistics in Geotechnics: Technical and Economic Risk Estimation, Austria, 39-46.

Low, B. K., and Tang, W. H. (1997). "Efficient reliability evaluation using spreadsheet." J. of Engrg. Mech., ASCE, 123, 749-752.

Popescu, R., Deodatis, G., and Nobahar, A. (2005). "Effect of random heterogeneity of soil properties on bearing capacity." Prob. Engrg. Mech., 20, 324-341.

Soubra, A.-H. (1999). "Upper-bound solutions for bearing capacity of foundations." J. of Geotech. \& Geoenv. Engrg., ASCE, 125(1), 59-68.

Vanmarcke, E. (1983). "Random Fields: Analysis and Synthesis." Published by MIT Press, Cambridge MA, 382p. 